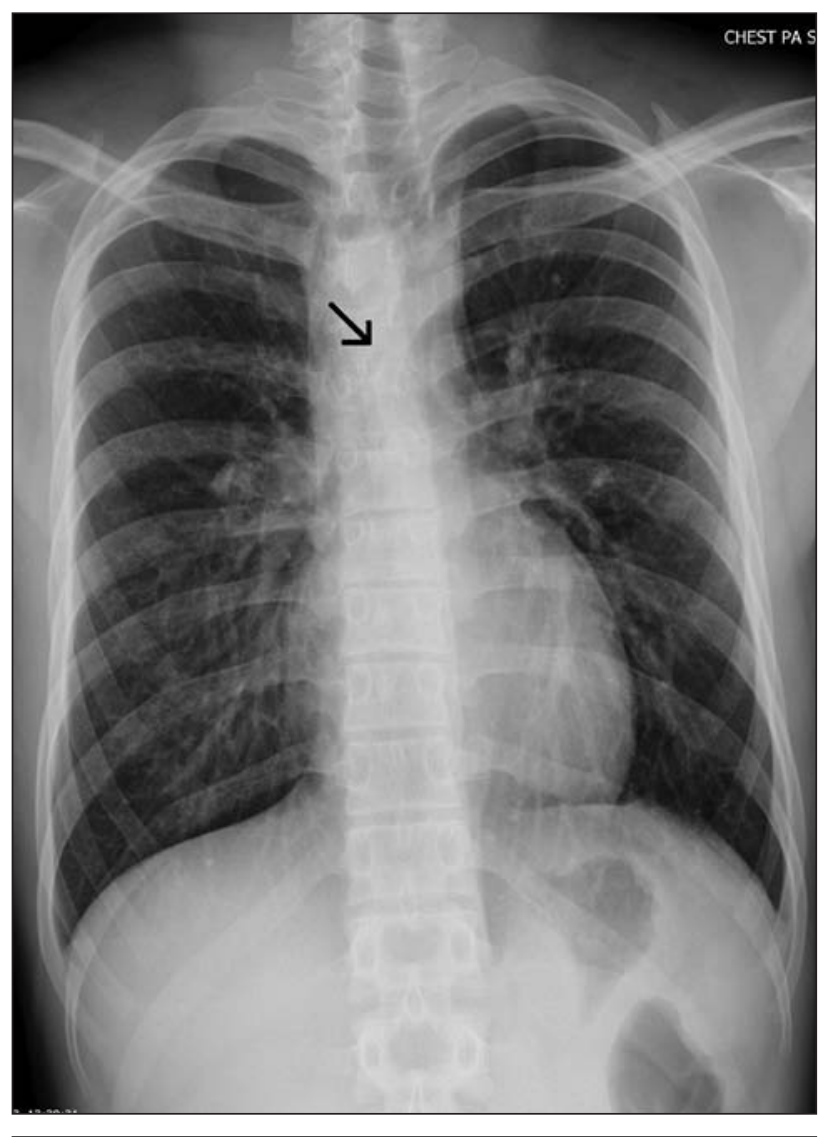

Figure 1: Chest radiograph of a 20-year-old man showing a right tracheal bronchus (arrow) and paratracheal mass with the trachea shifted to the left.

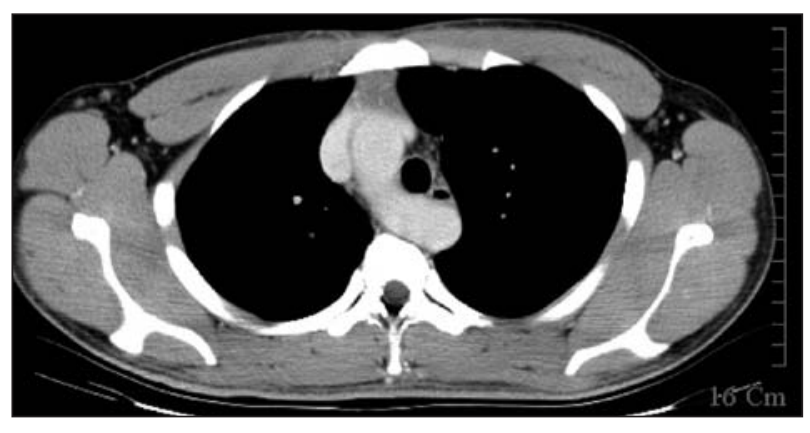

Figure 2: Computed tomography scan of the chest showing compression of the trachea by the right aortic arch.

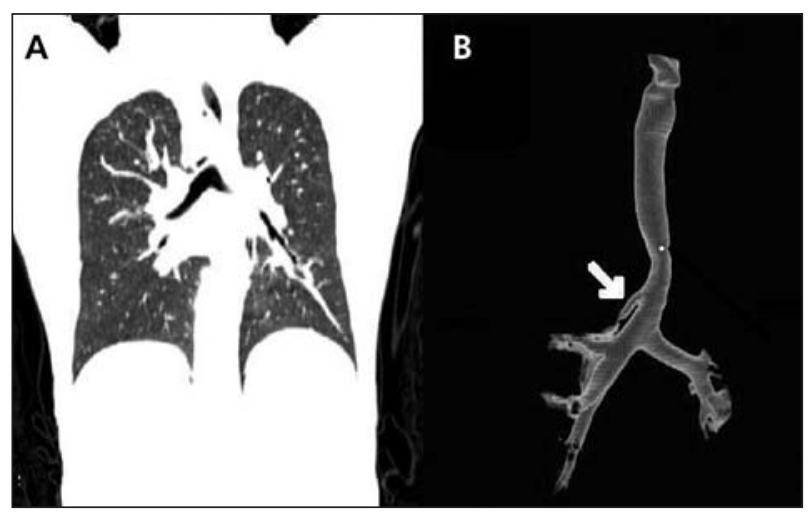

Figure 3: (A) A computed tomography scan in coronal view and (B) a 3-dimensional reconstruction of the scan show an abnormal bronchial structure (arrow) arising from the main bronchus above the carina.

\title{
Tracheal bronchus
}

A previously healthy 20 -year-old man presented with a 4-day history of intractable cough and fever. A chest radiograph showed a right tracheal bronchus and a right paratracheal mass with the trachea shifted to the left (Figure 1). A contrastenhanced computed tomography scan of the chest showed a right aortic arch and compression of the trachea (Figure 2). A 3-dimensional reconstruction of a computed tomography scan of the chest in coronal view was created to make the tracheal bronchus more visible (Figure 3). The patient's respiratory symptoms resolved after a course of oral antibiotic therapy.

First described by Sandifort in $1785,{ }^{1}$ tracheal bronchus is an aberrant, accessory or ectopic bronchial branch arising directly from the lateral wall of the trachea ${ }^{2}$ above the carina. It is congenital and has an incidence of about $2 \% .^{3}$ A right-sided bronchus such as the one we describe is not rare. Although developmental bronchial anomalies usually manifest in infancy or early childhood, some people may have no symptoms until adulthood. The anomaly may be discovered incidentally on computed tomography scans of the chest performed for some other reason. Tracheal bronchus is associated with recurrent infection and, in children, respiratory distress. Rarely, a tracheal bronchus may be intubated inadvertently during the administration of anesthesia or the treatment of respiratory failure. The resulting obstruction can cause atelectasis, postobstructive pneumonia or respiratory failure.
Fu-Chieh Shih MD

Wei-Jing Lee MD

Hung-Jung Lin MD

Department of Emergency Medicine

Chi-Mei Medical Center

Tainan, Taiwan

\section{REFERENCES}

1. Kubik S, Müntener M. Bronchus abnormalities: tracheal, eparterial, and pre-eparterial bronchi [German]. Fortschr Geb Rontgenstr Nuklearmed 1971;114:145-63.

2. Ghaye B, Szapiro D, Fanchamps JM, et al. Congenital bronchial abnormalities revisited. Radiographics 2001;21: 105-19.

3. O'Sullivan BP, Frassica JJ, Rayder SM. Tracheal bronchus: a cause of prolonged atelectasis in intubated children. Chest 1998;113:537-40. 